\title{
Taking Real-life to Cyberspace: Determining Factors Influencing Virtual Homophily among Members of Faith Communities on online Social Networks
}

\author{
Jacob Dankasa, \\ University of North Texas, Denton, USA
}

\begin{abstract}
Research on how members of faith communities interact on online social networks and the factors that influence their computer-mediated communication are lacking. This study applies the concept of homophily to investigate the factors that encourage the development of relationship ties among members of faith communities on online social network sites such as Facebook. A survey was conducted with 210 members of Catholic faith communities on Facebook. Findings revealed that active involvement with a real-life faith community predicts increased relationships and interaction among members on online social networks. Active involvement was found to be a major factor influencing virtual religious homophily. The findings support the notion that online social behavior reflects offline behavior. The study has several implications for the use of social media by religious organizations for building an online community of faith as an extension of the real-life church community.
\end{abstract}

Keywords -, Computer-mediated communication, faith community, homophily, online social networks, social network theory, social behavior on Facebook,

\section{INTRODUCTION}

Homophily is a concept of social network theory which holds that "a contact between similar people occurs at a higher rate than among dissimilar people" [1, p. 416]. It proposes that people drawn from a population or a similar network are more likely to be connected if they have matching characteristics [2]. Studies on homophily focus on finding the factors that encourage or influence the similarities that lead to the development of particular ties in a social network [3]. Several studies $[4,5]$ have been conducted to determine homophily on various social networks in different settings. While most studies of homophily have centered on investigating offline social networks, a few that have examined the concept on online environment $[6,7]$ have found support for homophily, while others did not [8].

Social network theory has been applied in numerous studies $[9,10]$; some of these studies have examined offline faith communities [11]. However, studies focusing on the application of social network theory to the interaction of members of faith communities on online social networks are lacking. The present study examines the concept of homophily and how it applies to the social behavior and communication among members of faith communities on online social networks such as Facebook.

Online social networks (OSNs) are "web-based services that allow individuals to (1) construct a public or semi-public profile within a bounded system, (2) articulate a list of other users with whom they share a connection, and (3) view and traverse their list of connections and those made by others within the system" [12, p. 211]. Online social networking is an aspect of social media that is mostly, but not exclusively, Internet-based communication [13]. Online social networking has become a very popular means of communication among many users in a variety of settings. Individuals, organizations and communities have turned to online social networks to enhance and consolidate the exchange of information and to foster relationships. Religious organizations are increasingly using online social networks. Many religious communities such as churches have pages and profiles on various OSNs that are designed for the spiritual, moral and intellectual growth of their members. In addition to the dedicated churches' pages on OSNs, members use their individual profiles to connect with other members, friends and families.

To date, the study of online social networks has concentrated on users from other settings such as college campuses $[14,15]$, healthcare centers [16, 17], and workplaces $[18,19]$. Previous studies of OSNs have shown that factors peculiar to users, such as personality traits, gender and age, influence or determine activities and behaviors on social networks [20,21]. Some studies have also shown that behaviors on OSNs are reflections of offline behavior [22, 23]. Despite the large volume of research that has been done on online social networks, very little has been done to study how members of faith communities interact on online social networks and what factors influence their behavior. 
This study applies the concept of homophily to investigate the factors that encourage the development of relationship ties among members of faith communities on online social networks. According to Kadushin [3], when examining homophily in a study, it is critical for the researcher to determine the characteristics, attributes or type of activities that are to be used in a given situation. Furthermore, Bisgin et al. [8], whose study of homophily in online social networks did not find support for the presence of homophily, recommended further research to expand the study beyond the two online communities they examined. Bisgin et al. [8, p. 536] proposed further research to investigate homophily "from different perspectives, identifying various factors that influence construction of new ties and their longevity in virtual and/or physical world." The present study responds to this need by looking into online social networks in a religious domain that has not been given much attention. It intends to find the matching characteristics of members of faith communities that influence their connection and interactions with one another on online social networks such as Facebook. This study particularly tested the social interactions of members of faith communities on Facebook against variables such as age, gender, spiritual experience and active involvement in a real-life church. Specifically, it examines which of these variables or factors is a better predictor of social behavior among members of faith communities on online social networks. The study attempts to answer the following questions:

RQ1: Is there a relationship between spiritual experience and social behavior on online social networks?

RQ2: Is there a relationship between active real-life faith community involvement and social behavior on online social networks?

RQ3: Is there a difference in the social behavior of members of faith communities on online social networks based on gender and age?

RQ4: If the possible effects of gender and age are controlled for, which is the best predictor of social behavior of members of faith communities on online social networks: spiritual experience or active community involvement?

Understanding the way members of faith communities use the online social networks through the lens of social network theory may help religious organizations fashion modalities to extend their faith community bond beyond their brick-and-mortar church buildings. The study may help our understanding of religious homophily on online social networks, and by extension, advance our understanding of social network theory and its application to online social networks of religious domain.

\section{SOCIAL NETWORK THEORY AND FAITH COMMUNITIES}

Social network theory provides concepts and propositions for studying social networks. "A network consists of a set of actors or nodes along with a set of ties of a specified type (such as friendship) that link them" $[24$, p. 2]. A network is made up of the actors and the relationships between or among the actors. The actors can be organizations or individuals that are referred to as the nodes. The actors and their relationships are described in terms of nodes and ties. The nodes are the people or organizations in the network and the ties are the relationships that link these people [3], and the relations of nodes in a network are described in terms of strong or weak ties [25]. Social network theory has been used to explain social relationships in various contexts [26, 27]. There are several social network theories, developed by different scholars [25, 28, 29]. The concept of homophily is particularly important to this study. Homophily is a concept introduced by Lazarsfeld and Merton [29] to describe social life in which people who are alike in certain characteristics associate with one another. It is a principle which proposes that similarity brings about connections. It holds that two people drawn from a population or a similar network are more likely to be connected if they have matching characteristics [2]. In other words, people form friendships and groups if they have similar characteristics. According to Lazarsfeld and Merton [29], there are two types of homophily: status homophily and value homophily. Status homophily is a type of similarity that can either be inherent—such as race, age, and sex-or acquired through marriage, education or religion. This happens especially with individuals who share similar social status, which influences them to associate with one another. Value homophily, on the other hand, happens among people who think alike due to certain beliefs or attitudes and not necessarily social status. The similarities they share may lead to fellowship even if they are of different social status. Several studies $[4,30]$ have used the concept of homophily to describe factors that lead to interactions and relationships in a network. Some of these studies have applied social network theory to examine the relationship between embeddedness in religious friendship networks and church activities [31, 32]. Stroope [33] found social embeddedness in a religious congregation to be a predictor of increased religious activities. One thing these studies have in common is their concentration on examining relationships in physical, offline faith communities. None of the studies examined online friendship networks of faith communities. In addition, the previous studies focused on investigating the influence of offline religious friendship networks on increased outcomes in the church activities of members. Most of the findings supported the notion that "social network theory suggests that embeddedness within a set of gratifying social relationships is an important predictor of religiosity" [34, p. 398]. 
While these studies found that participation in social networks of faith communities influences members to be active in their churches, there are only a few that examine the reverse of the findings: will active participation in offline faith communities influence social interactions among members? Lim and Putnam [11], for instance, found that religious people who attend religious events and services regularly and develop social networks with other members are more satisfied with their lives. In addition, they found that the development of strong relationships among congregation members depends on the strength of the religious identity. People become more connected to their religious social networks when they feel a strong sense of belonging to their religious community. The study suggests that people who are more involved in their faith communities feel the impact of the community's friendships in their lives. The study supported Ellison and George [35], who found that frequent church-goers, when compared to non-church-goers, make more friends outside their family networks and receive more social support within their church networks. This is because religious faith communities facilitate wide-reaching friendships as a result of the coming together of people with varying characteristics to share similar social and religious values. These studies were, however, all based on face-toface, offline faith community social networks.

At the moment, studies that examine factors influencing relationships among members of faith communities on online social networks are lacking. The present study intends to examine the influence of factors such as spiritual experience, active community involvement, age and gender on social interaction among members of faith communities on online social networks. The previous studies were more concerned with using relationships in social networks to build the real-life church community. This study, on the other hand, is interested in using the activities of members in the real-life faith community to propose the development of an extension of the faith community to online social networks in order to build online religious communities.

\section{COMPUTER-MEDIATED SOCIAL LIFE}

Computer-mediated social life (CMsl) is described in this study as those computer-mediated communications, interactions or activities that take place on online social networks such as Facebook, Twitter, LinkedIn, and Instagram. Online social networks are increasingly becoming acceptable means of communicating, developing relationships and networking [36]. Studies on the uses and users of OSNs are growing and emerging from different disciplines and areas of learning because computer-mediated communications (CMC) traverse boundaries. This is, in part, due to the growing interest in the use of OSNs, especially among adults. The number of adults using the Internet for social networking increased from $8 \%$ in 2005 to $35 \%$ by 2009 [37]. That number has doubled to $74 \%$ by 2014 [38].

Studies on how individuals or groups use OSNs have been well documented, studies and the relationship between offline and online behaviors has also been investigated in multiple studies [39, 40]. Researchers have asked various questions to understand the uses of OSNs by adults, their specific behavior, and the psychology behind their behaviors. The findings of these studies support the notion that social behaviors online reflect offline behavior [41, 42]. For instance, those who spent much time socializing offline are more likely to exhibit similar behavior on OSNs. These findings were corroborated by other studies [43], which found that users of OSNs maintain or consolidate relationships that are already existing offline rather than make unfamiliar new relationships. Gosling et al. [23] agreed that user behavior in online social networks is a reflection of one's social reality in the real world. However, they pointed out that the context of use determines the kind of traits manifested; some traits are more often manifested in some contexts than in others. For example, the context of use for college students may yield different results from another setting, such as the workplace. This demonstrates the need to study personality traits or behaviors of users on OSNs from a variety of contexts, including faith communities.

Studying OSNs in context is important, considering variations in personality traits of users. Some studies that have related personality traits to social network use have found that maintenance of offline ties on OSNs is dependent on factors such as the gender of the user. Muscanell and Guadagno [37] investigated individual differences in the use of social networks. They applied the Big 5 factor model of personality, examining its relationship to gender. The goal was to find how gender and personality determine the kinds of activities users carry out on online social networks. Muscannell and Guadagno [37] conducted an online survey of 238 undergraduate psychology students and found a positive relationship between gender and the personality of the user. Women were found to be more engaged in activities geared toward maintaining an already established offline relationship, while men were more likely to use the sites for networking, finding friends and dates, and making new relationships. These findings lend credence to studies that have found men to be more adventurous [44], while concern for privacy and personal safety could cause women to be less likely to disclose their identity and relate with strangers on OSNs [45, 46].

Computer-mediated social life may have benefits for users, depending on the gratifications derived. According to Antoci, Sabatini, and Sodini [47], online social interactions on sites such as Facebook and Twitter are less time-consuming than face-to-face meetings. They allow for interactions without the limit of 
Taking Real-Life to Cyberspace: Determining Factors Influencing Virtual Homophily...

geographical distance and enable the user to accumulate more social capital, since they allow social interactions through viewing and posting on another's profile even when the other user is offline. Facebook, for instance, has functionalities that allow users to show their identity, to have conversations with other users, and to share content and be accessible to others by showing their presence and availability in virtual worlds. It also has functions that allow for the creation of relationships, the building of reputations and the setting up of groups that are representative of communities of interests [48]. These features of OSNs, according to Antoci et al. [47], will reduce the effect of an obsessively busy society that causes people to be less involved with relational activities and to disengage from real-life community participation. Such disengagements have led to a decline of social capital in real life [49]. This points to the need for understanding the social interactions of members of faith communities on online social networks in order to sustain and enhance the community spirit in virtual space in an attempt to complement the already-developed relationships in the real-world church community.

\section{METHODOLOGY}

The study was a survey conducted with Facebook users who were members of Catholic faith communities in Dallas, Texas, U.S.A. A questionnaire was developed and a link provided on the Facebook pages of several Catholic parishes and the private timelines of some Facebook users.

\section{Participants}

A total of 210 members of Catholic parishes in Dallas participated in this study. They were $23.8 \%$ males and $76.2 \%$ females. Among the participants, $15.2 \%$ were ages $18-29 ; 41.4 \%$ were ages $30-49 ; 29.5 \%$ were 50-64 years old, and $13.8 \%$ were 65 years or older. The ethnicity of the participants was reported to be 72.9\% Caucasian, $17.6 \%$ Hispanic or Latino, 3.3\% Asian, 2.9\% African or African American, $1.4 \%$ Native American, and $1.9 \%$ other ethnicity. A majority of the participants (91\%) reported that their church communities have a Facebook page, 1.9\% said their churches did not have Facebook page, and $7.1 \%$ said they did not know whether their church communities have a Facebook page or not.

On their use of Facebook, 84.8\% reported that they belong to their church community's Facebook page, $14.3 \%$ said they do not belong to their church's Facebook page, and 1\% were missing data because they did not answer the question. Among the participants, 39.5\% spend less than an hour daily on Facebook, $41.9 \%$ spend 1 to 2 hours daily, $14.3 \%$ spend 3 to 4 hours, $3.3 \%$ spend 5 to 6 hours, and $1 \%$ spend more than 6 hours daily using Facebook.

\section{Measures}

\section{Social Behavior on Facebook}

The social behavior of participants was measured using a six-item scale of questions on varieties of activities on Facebook. The questions included: I read the post on my church Facebook page; I have commented or liked a post on my church Facebook page; I have commented or liked a profile post of a member of my church community on Facebook; I have communicated and related with members of my church community on Facebook. The frequency with which they engage in these activities was assessed on a scale ranging from 1 (never) to 5 (always). The last two questions in the scale: How many of your friends on Facebook are members of your church community? If you have friends on Facebook who are also members of your church community, how many of them do you also know in real-life church community? were assessed on a scale ranging from 1 (none) to 5 (all). This social behavior scale was developed and used for the first time in this study. The scale has an acceptable internal consistency with Cronbach alpha coefficient of .79, $($ mean $=17.98, S D=4.91)$.

\section{Spiritual Experience}

Spiritual experience of participants was measured using a 16-item Daily Spiritual Experience Scale (DSES) derived from Underwood [50]. The Daily Spiritual Experience Scale (DSES) is designed to measure ordinary experiences concerning how people relate to, and become aware of, the divine or the transcendent in their daily lives. Examples of questions include: I feel God's presence; I find strength in my religion or spirituality; I feel God's love for me directly. The responses of the scale in the present study were reversed, with higher numbers indicating higher spiritual experience. The responses ranged from 1 (Never) to 6 (Many times a day). The original scale has higher numbers indicating lower spiritual experience. One item in the scale: In general, how close do you feel with God, was not reversed and the response ranged from 1 (Not at all close) to 4 (As close as possible). According to Underwood and Teresi [51], the Daily Spiritual Experience Scale has good internal consistency, with a Cronbach alpha coefficient reported of .95. In the present study, the Cronbach alpha coefficient was .93 , $($ mean $=70.81, S D=12.50)$.

\section{Community Involvement}


Taking Real-Life to Cyberspace: Determining Factors Influencing Virtual Homophily...

Using a response scale ranging from 1 (Not at all involved) to 5 (Very much involved), one item in the survey measured individual participants' involvement in a real-life faith community. The question asked: How involved are you with ministries/activities in your church community? (mean $=3.85, S D .=1.27)$.

\section{RESULTS}

\section{Relationship Between Spiritual Experience and Online Social Behavior}

To answer the first research question, the relationship between spiritual experience (measured by the DSE scale) and social behavior of participants on Facebook (measured by the social behavior scale) was investigated using the Pearson product-moment correlation coefficient. There was a weak positive correlation between the two variables, $r=.16, n=207, p=.03$. This indicated that a high level of spiritual experience was associated with a high level of social interaction on Facebook. It showed that members who score high on spiritual experience are more likely to interact more with other community members on Facebook. This relationship was, however, weak.

\section{Relationship Between Church Community Involvement and Online Social Behavior}

To find the relationship between church community involvement and social behavior on online social networks, Spearman's Rho correlation coefficient was used to analyze the data. The results showed a moderate positive correlation between the two variables, rho $=.47, n=207, p=.00$, with a high level of faith community involvement associated with a high level of interaction with other members on Facebook. This indicates that members who are more involved in their church community tend to relate more with other members on Facebook. This answers the second research question.

\section{Differences Based on Gender and Age}

In order to determine whether there was a difference in the social behavior of the participants on Facebook based on their gender, an independent-samples $t$-test was conducted. The results showed no statistically significant difference in the scores for males $(M=18.06, S D=4.67)$ and females $(M=17.95, S D=$ $5.0 ; t(205)=.14, p=.89$, two-tailed). This shows that the social behavior of the participants on Facebook is the same for both males and females.

A one-way between-groups analysis of variance was conducted to explore whether there was a difference in the social behavior of participants based on their age. The participants were distributed into four groups based on their age (Group 1: ages 18-29; Group 2: ages 30-49; Group 3: ages 50-64; Group 4: 65 years and older). There was no statistically significant difference found among the four age groups: $F(3,203)=1.46$, $p=.23$. This indicates that the participants' social behavior on Facebook is the same regardless of their age differences.

\section{Predictor of Social Behavior on OSNs}

Spiritual experience, faith community involvement, age and gender were used as independent variables to predict the social behavior of participants on Facebook. Standard multiple regression was carried out to determine the effect of these four independent variables on the online social behavior of participants. This was to determine the independent variable that is a better predictor of social behavior on Facebook between spiritual experience and active community involvement, controlling for the possible effects of gender and age. The model was found to be statistically significant $(F(2,204)=22.51, p=00)$, which shows that it is unlikely that the results occurred by chance. The $R^{2}$ indicates $24.2 \%$ of the variance in the scores of the online social behavior can be explained by variance in the four predictor variables. The findings suggested that active faith community involvement $(\beta=.47)$ was more of a predictor of the online social behavior of participants than spiritual experience $(\beta=.09)$. Age and gender were the least predictive of the online social behavior of participants (see Table 1). Active church community involvement $(t=7.50, p=.00)$ was shown to be a statistically significant predictor of the online social behavior of participants, while spiritual experience $(t=1.42, p=.16)$, gender and age were not statistically significant predictors. Active church community involvement alone explains $21 \%$ of the variance in the total scores of online social behavior, out of the $24.2 \%$ total $R^{2}$ value for the model (part = .459). This answers the fourth research question.

Table 1. Regression model with social behavior as the criterion variable.

\begin{tabular}{llll}
\hline Variables & $\beta$ & $\mathrm{t}$ & $\mathrm{SE}$ \\
\hline Spiritual experience & .09 & 1.42 & .03 \\
Active community involvement & $.47^{*}$ & 7.50 & .24 \\
Age & -.08 & -1.24 & .33 \\
Gender & .01 & .18 & .71 \\
\hline Note: ${ }^{*} \mathrm{p}<.05$ & & &
\end{tabular}


Taking Real-Life to Cyberspace: Determining Factors Influencing Virtual Homophily...

\section{DISCUSSION}

The findings of this study revealed that members of faith communities actively use online social networks (OSNs) such as Facebook to relate among themselves. Their spiritual experience as members of religious communities and their involvement in their faith communities were both significantly related to how they interact and socialize with members of the same community on Facebook. The findings indicated that members of faith communities with a high level of spiritual experience and those who are actively involved in their real-life faith communities are more likely to connect with the OSNs of their faith communities and to interact with other members online. While both variables showed a statistically positive relationship with social behavior on Facebook, it was only a weak relationship for spiritual experience, but a moderate relationship for community involvement.

When the effects of age and gender were controlled for in the model, the results showed that spiritual experience did not reach statistical significance, while active community involvement was statistically significant. This indicates that active involvement with a real-life church community is more likely than spiritual experience to predict increased relationship and interaction among members of faith communities on OSNs. The results of the multiple regression showed active involvement with the activities of a faith community as the only significant predictor of members' social interaction on Facebook. Spiritual experience, gender and age were not statistically significant predictors. This suggests that when members of faith communities actively participate in the activities of their real-life brick-and-mortar churches they are more likely to build relationships with other members. These relationships are translated into social interactions on online social networks among members.

These results show that active involvement in real-life church activities or ministries is the most likely predictor of religious homophily of members of faith communities on online social networks. The findings of this study agree with McPherson [1, p. 428] that "people who are more structurally similar to one another are more likely to have issue-related interpersonal communication and to attend to each other's issue positions, which, in turn, leads them to have more influence over one another." Those who are actively involved in their church community tend to relate among themselves in a deeper level by sympathizing, comforting, advising and sharing family and personal problems with one another on online social networks. The findings are also in line with Gosling et al. [23] and Weisbuch et al. [42] that online social behavior reflects offline behavior. It also supports Ellison et al. [43], who found that users of online social networks maintain or consolidate relationships that are already existing offline. The type of ties found here seems to fall within the category of induced homophily resulting from random mixing within the faith community [52]. Here, the gender, age, geographic distance and family ties of members did not hinder relationship among members. This affirms that their religious affiliation is the uniting factor that extends to online relationships, which demonstrates the presence of virtual religious homophily among members of these faith communities.

There was no difference in the way the members of these faith communities behave on Facebook based on their gender and age. The behaviors of members were found to be the same for both males and females across all ages. This was contrary to Muscanell and Guadagno [37], who found women to be more engaged in activities geared towards maintaining an already established offline relationship than men. It is likely that in the case of these participants, due to the faith community experience of members, both men and women tend to behave the same way in trying to maintain faith-based relationships online.

The findings of the current study suggest that describing the behavior of individuals or groups of people based on their religious beliefs will depend on different factors, particularly the characteristics of the participants. Participants bonded to their faith communities may likely show different behaviors among members in relation to their religious beliefs than those not committed to real-life faith communities. It appears that it takes more than being spiritual to connect with faith communities on online social networks. Active faith community involvement is an important factor in enhancing relationships among members of faith communities on OSNs, as the findings of the present study show.

These findings support the notion that users of the Internet should be studied according to their context and the culture of use. Thorne [13, p. 9] believes that "Internet communication tools, like all human creations, are cultural tools that carry interactional and relational associations, preferred uses (and correspondingly, inappropriate uses), and expectations of genre-specific communicative activity." Hence, studying not just individual users, but how communities and groups use the tools of the new technologies within their specific cultural and social contexts, is very important, since such studies may reveal further that the Internet is not a neutral, but a situational, medium. In other words, the Internet-mediated technology is, in and of itself, a culture whose uses and meanings vary according to the community of users [53]. The present study contributes to the culture of use of the new communications technology tools from the perspective of the faith community.

From the demographic characteristics of the sample in this study, there was evidence of an increased presence of older members of faith communities on OSNs such as Facebook. A large number of the participants, a combined total of $43.3 \%$ in the study, were 50 years or older. Only $15.2 \%$ were $18-29$ years old, while $41.4 \%$ were 30-49 years old. Although the social behaviors of these faith community members on Facebook do not differ, it shows that older adults in faith communities are likely to be interested in developing faith-based 
Taking Real-Life to Cyberspace: Determining Factors Influencing Virtual Homophily...

community relationships online. The presence of older adults on Facebook, as found in the present study, is consistent with previous studies that found an increased use of communications technologies by older adults $[54,55]$.

\section{IMPLICATIONS OF FINDINGS}

This study has several implications for the use of online social networks by religious organizations to build an online community of faith as an extension of the real-life church community. The study aligns with Lim and Putnam [11], who found in their study that religious people who reported that they were very satisfied with their lives tended to be those who attend religious services regularly and build social networks in their communities. Members of faith communities relate to one another in their real-life church communities through participation in different ministries. These ministries are small communities within the larger faith community where members not only share their spiritual journeys, but also build friendship ties with one another. Online social networks such as Facebook may provide a medium for members to widen their community ties and relate to other members online. Members can seek help online from one another, serve as caregivers through words of comfort, and share and identify with one another. The spiritual growth of members is of value to any faith community, but the large population size of some churches makes it difficult for some members to be physically connected with their faith communities. Hence, online social networks may provide a good opportunity to extend spiritual care beyond bricks-and-mortar church communities. Creating homophilous ties on online social networks blurs the geographic distance between active members and provides an opportunity for them to have anytime, anywhere communication with one another outside the physical meeting space and time.

Kietzmann et al. [48, p. 249] suggest that "firms should develop strategies for monitoring, understanding, and responding to different social media activities." Religious organizations should not be left out in this venture. This points to the need to study the social media use of faith communities in order to understand how social media can be used effectively to serve the religious needs of users in a time when all demographics are present online. This study may be of significance to religious organizations which maintain a virtual community as an extension of real-world church communities. Thus they can minister to their members anytime, anywhere, communicating religious information and keeping members involved in the lives of other members, thus enhancing their spiritual growth.

As this study shows, the presence of older members of faith communities on online social networks is an opportunity for ministry in religious organizations. There may be a need to be attentive to the way older adults interact online, because computer-mediated communication (CMC) was found to reduce the social isolation of older people. Ofei-Dodoo, Nilsen, and Smith [56] found that older persons who communicated with family through CMC developed the desire to communicate more with computers; they reported that they were less lonely, as a result, than those who do not use computers. This presents a significant opportunity for faith communities to extend spiritual care to their older members using online social networks.

Building faith-based communities is central to religion. Online social networks are assets which religious organizations cannot afford to ignore. Faith communities should view online social networks with the kind of seriousness that marketing and business firms give to these media. The findings of this study may help faith communities in developing communication strategies to meet the spiritual needs of their members not only in offline, real-life community settings but also in cyberspace. The study paves the way for the development of a suitable model for online religious communication and community engagement using the social media, especially the online social networks. It provides a framework for future studies of social media use in religious settings, and restates the growing influence of new media beyond the realm of business and academia.

This study has some limitations, especially in its reliance on self-report measures, which could raise some validity issues on the authenticity of the reported behaviors. Further studies may collect data from observed behaviors based on the activities and communications of the sample on Facebook or other social networking sites. This study was conducted only among members of the Catholic church in Dallas using Facebook as a platform. This may affect the generalization of the findings to other religious denominations, other geographical locations or other social media platforms. Future research may be conducted among users of Facebook or other online social networks who are members of other Christian faith communities, such as an Evangelical denomination, and compare those findings with the present study of members of Catholic faith communities. Nevertheless, the findings present us with indications of how a sample of members of religious communities use the online social networks to enhance relationship from their real-life faith communities.

\section{CONCLUSION}

This study contributes to the understanding of the impact of active community involvement on the computer-mediated social life (CMsl) of members of faith communities. It shows that members of faith communities use OSNs to communicate and interact among themselves. Active involvement or participation in real-life faith communities was found to be a better predictor of virtual religious homophily among members of 
Taking Real-Life to Cyberspace: Determining Factors Influencing Virtual Homophily...

faith communities than spiritual experience, age or gender. Members who are more involved with their faith communities are more likely to interact with other members on online social networks, and to connect with activities on their churches' Facebook pages. The study contributes to the literature on social media from the context of religious communities, and on the use of information and communication technologies for building online faith-based communities as an extension of real-life faith communities. It adds to our knowledge of religious homophily on online social networks and enhances our understanding of social network theory as it applies to the use of online social networks from the perspective of faith communities. Religious organizations can utilize the online social networks to enhance and consolidate the exchange of information, and to foster relationships by providing members with more avenues connecting them with one another and with their faith communities.

About the Author:

Jacob Dankasa holds a master's degree in Mass communications from St. Cloud State University Minnesota, USA and a PhD in Information Science from University of North Texas, Denton, USA. He can be contacted at jdankasa@gmail.com.

\section{REFERENCES}

[1] J. M. McPherson, L. Smith-Lovin, and J.M. Cook, Birds of a feather: Homophily in social networks. Annual review of sociology, 27, 2001, 415-444.

[2] L.M. Verbrugge, The structure of adult friendship choices. Social forces, 56(2), 1977, 576-597.

[3] C. Kadushin, Understanding social networks: Theories, concepts, and findings. (New York: Oxford University Press, 2012).

[4] G. Kossinets and D.J. Watts, Origins of homophily in an evolving social network1. American journal of sociology, 115(2), 2009, 405-450.

[5] H. Louch, Personal network integration: transitivity and homophily in strong-tie relations. Social networks, 22(1), 2000, 45-64.

[6] H. Lauw, et al., Homophily in the digital world: A LiveJournal case study. IEEE Internet Computing, $14(2), 2010,15-23$.

[7] M. Thelwall, Homophily in myspace. Journal of the American Society for Information Science and Technology, 60(2), 2009, 219-231.

[8] H. Bisgin, N. Agarwal, and X. Xu, Investigating homophily in online social networks. Proceedings of the IEEE/WIC/ACM International Conference on Web Intelligence and Intelligent Agent Technology (WIIAT), USA, 2010, 533-536.

[9] K. Flaherty, et al., Social network theory and the sales manager role: engineering the right relationship flows. Journal of Personal Selling \& Sales Management, 32(1), 2012, 29-40.

[10] J. Worrell, M. Wasko, and A. Johnston, Social network analysis in accounting information systems research. International Journal of Accounting Information Systems, 14(2), 2013, 127-137.

[11] C. Lim and R.D. Putnam, Religion, social networks, and life satisfaction. American Sociological Review, 75(6), 2010, 914-933.

[12] D.M. Boyd and N.B. Ellison, Social Network Sites: Definition, History, and Scholarship. Journal of computer-mediated communication, 13(1), 2008, 210-230.

[13] S.L. Thorne, Computer-Mediated Communication, in N. Hornberger \& N.V. Duesen-Scholl (Eds.), Encyclopedia of language and education, (New York: Springer, 2008) 325-336.

[14] A. Holman and A. Sillars, Talk about "hooking up": The influence of college student social networks on nonrelationship sex. Health communication, 27(2), 2012, 205-216.

[15] M.A. Moreno, et al., Feeling bad on Facebook: depression disclosures by college students on a social networking site. Depression and anxiety, 28(6), 2011, 447-455.

[16] J.S. Li, et al., Approaches to the prevention and management of childhood obesity: the role of social networks and the use of social media and related electronic technologies a scientific statement from the American Heart Association. Circulation, 127(2), 2013, 260-267.

[17] S. Young, G. Szekeres, and T. Coates, Sexual risk and HIV prevention behaviours among AfricanAmerican and Latino MSM social networking users. International journal of STD \& AIDS, 24(8), 2013, 643-649.

[18] M.M. Skeels and J. Grudin. When social networks cross boundaries: a case study of workplace use of facebook and linkedin. Proceedings of the ACM 2009 international conference on Supporting group work, USA, 2009, 95-104.

[19] J. Vitak, et al. Why won't you be my Facebook friend?: Strategies for managing context collapse in the workplace. Proceedings of the iConference, USA, 2012, 555-557. 
Taking Real-Life to Cyberspace: Determining Factors Influencing Virtual Homophily...

[20] R.E. Guadagno, et al., Even in virtual environments women shop and men build: A social role perspective on Second Life. Computers in Human Behavior, 27(1), 2011, 304-308.

[21] E. Morris, She "Likes" it, He Doesn't: Gender Differences in Facebook Communication Behaviors, doctoral diss., University of Colorado: Boulder, 2013.

[22] M.D. Back, et al., Facebook Profiles Reflect Actual Personality, Not Self-Idealization. Psychological Science, 21(3), 2010, 372-374.

[23] S.D. Gosling, et al., Manifestations of personality in online social networks: Self-reported Facebookrelated behaviors and observable profile information. Cyberpsychology, Behavior, and Social Networking, 14(9), 2011, 483-488.

[24] S.P. Borgatti, and D.S. Halgin, On network theory. Organization Science, 22(5), 2011, 1168-1181.

[25] M.S. Granovetter, The strength of weak ties. American journal of sociology, 78(6), 1973, 1360-1380.

[26] W.R. Penuel, et al., Using social network analysis to study how collegial interactions can augment teacher learning from external professional development. American Journal of Education, 119(1), 2012, 103-136.

[27] T.J. Rowley, Moving beyond dyadic ties: A network theory of stakeholder influences. Academy of management Review, 22(4), 1997, 887-910.

[28] R.S. Burt, Structural holes: The social structure of competition. 1992, (MA: Harvard University Press, 1992).

[29] P.F. Lazarsfeld and R.K. Merton, Friendship as a social process: A substantive and methodological analysis. Freedom and control in modern society, 18(1), 1954, 18-66.

[30] S. Currarini, M.O. Jackson, and P. Pin, An economic model of friendship: Homophily, minorities, and segregation. Econometrica, 77(4), 2009, 1003-1045.

[31] C. Kadushin and L. Kotler-Berkowitz, Informal social networks and formal organizational memberships among American Jews: Findings from the National Jewish Population Survey 2000-01. Sociology of Religion, 67(4), 2006, 465-485.

[32] T.F. Martin, J.M. White, and D. Perlman, Religious socialization: A test of the channeling hypothesis of parental influence on adolescent faith maturity. Journal of Adolescent Research, 18(2), 2003, 169-187.

[33] S. Stroope, Social Networks and Religion: The Role of Congregational Social Embeddedness in Religious Belief and Practice. Sociology of Religion, 73(3), 2012, 273-298.

[34] J.C. Cavendish, M.R. Welch, and D.C. Leege, Social Network Theory and Predictors of Religiosity for Black and White Catholics: Evidence of a" Black Sacred Cosmos"? Journal for the Scientific Study of Religion, 37(3), 1998, 397-410.

[35] C.G. Ellison and L.K. George, Religious involvement, social ties, and social support in a southeastern community. Journal for the scientific study of religion, 33(1), 1994, 46-61.

[36] P.M. Valkenburg and J. Peter, Social consequences of the internet for adolescents a decade of research. Current Directions in Psychological Science, 18(1), 2009, 1-5.

[37] N.L. Muscanell and R.E. Guadagno, Make new friends or keep the old: Gender and personality differences in social networking use. Computers in Human Behavior, 28(1), 2012, 107-112.

[38] Pew Research Center, Social Networking Fact Sheet, 2014 [retrieved 2015 February 23]; Available from: http://www.pewinternet.org/fact-sheets/social-networking-fact-sheet/

[39] A.N. Joinson, Looking at, looking up or keeping up with people?: motives and use of facebook. Proceedings of the SIGCHI conference on Human Factors in Computing Systems, USA, 2008, 10271036.

[40] K. Subrahmanyam, et al., Online and offline social networks: Use of social networking sites by emerging adults. Journal of Applied Developmental Psychology, 29(6), 2008, 420-433.

[41] C. Lampe, N.B. Ellison, and C. Steinfield, Changes in use and perception of Facebook. Proceedings of the ACM conference on Computer supported cooperative work, USA, 2008, 721-730.

[42] M.Z. Weisbuch, Z. Ivcevic, and N. Ambady, On being liked on the web and in the "real world": Consistency in first impressions across personal webpages and spontaneous behavior. Journal of Experimental Social Psychology, 45(3), 2009, 573-576.

[43] N.B. Ellison, C. Steinfield, and C. Lampe, The benefits of Facebook "friends:" Social capital and college students' use of online social network sites. Journal of Computer-Mediated Communication, 12(4), 2007, 1143-1168.

[44] V.S. Helgeson, Relation of agency and communion to well-being: Evidence and potential explanations. Psychological Bulletin, 116(3), 1994, 412-428.

[45] A. Acquisti and R. Gross, Imagined Communities: Awareness, Information Sharing, and Privacy on the Facebook, in G. Danezis and P. Golle (Eds.), Privacy Enhancing Technologies, (Berlin: Springer Berlin Heidelberg, 2006) 36-58.

[46] J. Fogel and E. Nehmad, Internet social network communities: Risk taking, trust, and privacy concerns. Computers in human behavior, 25(1), 2009, 153-160. 
Taking Real-Life to Cyberspace: Determining Factors Influencing Virtual Homophily...

[47] A. Antoci, F. Sabatini, and M. Sodini, See you on Facebook! A framework for analyzing the role of computer-mediated interaction in the evolution of social capital. The Journal of Socio-Economics, 41(5), 2012, 541-547.

[48] J.H. Kietzmann, et al., Social media? Get serious! Understanding the functional building blocks of social media. Business horizons, 54(3), 2011, 241-251.

[49] D.L. Costa and M.E. Kahn, Understanding the American Decline in Social Capital, 1952-1998. Kyklos, 56(1), 2003, 17-46.

[50] L.G. Underwood, Ordinary spiritual experience: Qualitative research, interpretive guidelines, and population distribution for the Daily Spiritual Experience Scale. Archive for the Psychology of Religion, 28(1), 2006, 181-218.

[51] L.G. Underwood and J.A. Teresi, The daily spiritual experience scale: Development, theoretical description, reliability, exploratory factor analysis, and preliminary construct validity using health-related data. Annals of Behavioral Medicine, 24(1), 2002, 22-33.

[52] J.M. McPherson and L. Smith-Lovin, Homophily in voluntary organizations: Status distance and the composition of face-to-face groups. American sociological review, 52, 1987, 370-379.

[53] S.L. Thorne, Artifacts and cultures-of-use in intercultural communication. Language Learning \& Technology 7(2), 2003, 38-67.

[54] M. Madden, Older adults and social media, 2010 [retrieved 2015 February 20]; Available from: : http://www.pewinternet.org/Reports/2010/Older-Adults-and-Social-Media/Report.aspx.

[55] S. Sayago, D. Sloan, and J. Blat, Everyday use of computer-mediated communication tools and its evolution over time: An ethnographical study with older people. Interacting with Computers, 23(5), 2011, 543-554.

[56] S. Ofei-Dodoo, K.M. Nilsen, and R. Smith. Exploring older persons use of computer-mediated communication technologies. Proceedings of the 9th Annual Symposium: Graduate Research and Scholarly Projects, 2013. Wichita: Wichita State University. 